\title{
Novel Fuzzy Torque Vectoring Controller for Electric Vehicles with per-wheel Motors
}

\author{
Alberto Parra ${ }^{a}$, Martín Dendaluce ${ }^{a}$, Asier Zubizarreta ${ }^{b}$, Joshué Pérez ${ }^{a}$. \\ ${ }^{a}$ Tecnalia Research \& Innovation, Industry and Transport Division, Spain \\ ${ }^{b}$ Dept. of Automatics and System Engineering, University of the Basque Country (UPV/EHU) \\ Faculty of Engineering of Bilbao, Spain
}

\begin{abstract}
The continuous innovation on the electric vehicle area has led to the use of multi-motor powertrain topologies. These powertrains imply new degrees of freedom and better controllability, thus enabling elaborate control solutions for enhanced vehicle dynamics. This allows not only to achieve better vehicle stability, but also greater performance and responsiveness, leading to safer and more satisfying vehicle handling. This work presents a Fuzzy Torque Vectoring Controller for a rear-wheel drive car with independent motors per wheel. The developed design has been validated in a high fidelity vehicle dynamics simulation platform demonstrating its capability to enhance not only curve performance, but also stability.
\end{abstract}

Keywords: Torque Vectoring (TV), Electric Vehicle (EV), yaw rate, sideslip angle, Fuzzy Logic .

\section{INTRODUCTION}

The need of reducing global warming, air pollution and oil dependency has motivated not only the implementation of renewable energies, but also other technologies such as electric vehicles [1]. Although these types of vehicles are still not widely established, the sales of vehicles with electrified powertrains (fully-electric as well as hybrids) are increasing, and they have become one of the main research areas in the automotive industry. The integration of electric motors in propulsion systems provide not only better energy efficiency and lower pollution, but also increased controllability, as these motors offer better response time. This enables to achieve enhanced vehicle handling, not only in the longitudinal dynamics, but also in lateral dynamics. The potential of these aspects, together with increased degrees of freedom, are fuelling a notable interest in the field of control engineering.

Electrified propulsion systems offer a wide variety of possible topologies, with a notable diversity of hybrid solutions. Reducing the scope to purely electric vehicles, as targeted in this paper, the possible topologies can range from a single electric motor with a mechanical differential, to individual motors per wheel. The solutions with individually controllable motors offer the greatest potential for enhancements to the vehicle's dynamic handling. For this purpose, specialized algorithms, such as Torque Vectoring algorithms are used to control the torque on each wheel. These approaches are capable of improving not only the cornering capabilities (such as greater speed, lateral acceleration and overall responsiveness) but also the vehicle stability under certain circumstances.

Several strategies can be used to control the torque distribution in a Torque Vectoring approach, being most of them based on controlling the moment along the vertical axis of the vehicle (yaw moment). For this purpose, three main strategies are used [16]: the first one is based on the distribution of the torque in the rear axle; the second one is using active vehicle roll control systems to vary the lateral load distribution; finally, the third strategy consists in implementing a steering system in both axles. This work focuses on torque distribution strategies, as a rear-wheel drive vehicle has been selected as study case.

The torque distribution approaches have been implemented using a wide variety of control algorithms. Classical PID approaches have been analyzed in [6], where a very detailed comparison is made between two conventional controllers (traditional PID and adaptive PID) and more innovative controllers (sub-optimal Second Order Sliding Mode (SOSM) controller and twisting SOSM controller). The obtained results allow to deduce that no gain scheduling is necessary to achieve good performance. Furthermore, a better tracking in steady state is achieved for the suboptimal sliding mode approach but undesirable oscillations appear in the yaw rate during step maneuvers at high steering amplitudes. In conclusion, predictable behaviour and good frequency response are key characteristics in favour of PID controllers for real vehicle applications.

Other authors have proposed using advanced control approaches such as MPC [17] or integral 
sliding mode control [9]. Although these strategies provide good results, the computational cost of these approaches is higher than simpler approaches such as the PID-based ones.

Finally, some authors have proposed to use intelligent approaches, such as Neural Networks [10] or Fuzzy logic systems [11], demonstrating, though several manoeuvres, how torque vectoring controllers based on this last control technique improves vehicle's handling, stability and pathfollowing. In addition, the use of a fuzzy logic controller provides good results at low computational cost in comparison with previous approaches, being a good alternative to the implementation of torque distribution approaches.

Due to this, in this paper a novel Torque Vectoring algorithm based on fuzzy logic is proposed.The proposed approach provides enhanced lateral dynamic performance by reducing the sideslip angle and controlling the yaw moment at the same time. The approach has been validated in a rear-wheel driven vehicle implemented on a high fidelity dynamics simulator.

The rest of the paper is divided as follows. In section 2 the mathematical model for the generation of the reference variable for the developed fuzzy controller is explained. Section 3 describes the followed process for the design of this controller, which will be validated through the platform described in section 4 by comparing the fuzzy controller and a vehicle without this control system. Finally, the section 5 summarizes the most important ideas and future work.

\section{YAW RATE REFERENCE GENERATOR}

The developed torque distribution control approach is based on the control of the yaw moment of the vehicle, so that an appropriate yaw rate reference is required for proper performance of the controller. This section provides a detailed description of the model used for the yaw rate reference calculation in the controller. It must be noted that this model of reduced complexity is exclusively used for real-time execution inside the controller.

For the calculation of the desired yaw rate reference, the model known as "bycicle model" will be used (see Fig. 1), which provides a good balance between accuracy and computational cost. Some additional simplifications are done for the sake of computational performance: the center of gravity is assumed to be at a height of zero; the variation of the vertical force of each tire will not be taken into account; small slip angles assumption will be considered, so that $\sin (\beta)=\beta$ and $\cos (\beta)$ $=1$ (linear region): and the coefficient of lateral stiffness of the tire will be constant, defined by the ratio of the lateral force to the slip angle.

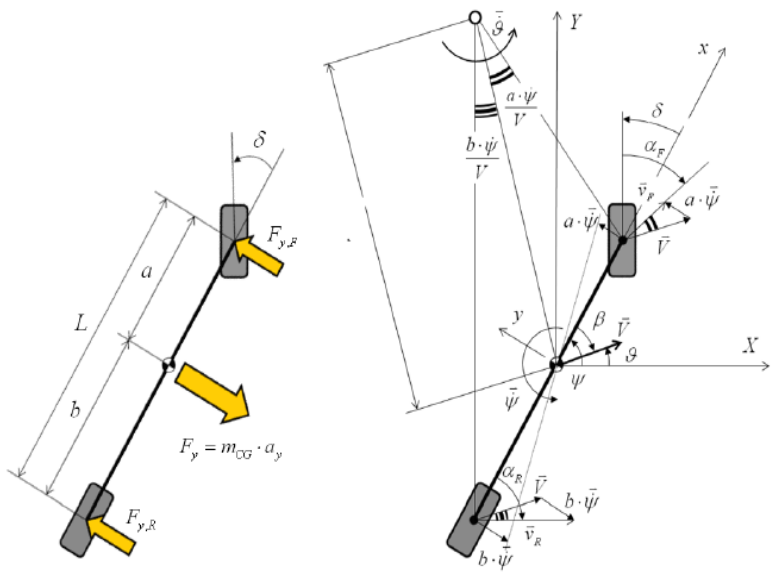

Figure 1: Bicycle model [13].

So, the desired yaw rate is given by the following equation:

$$
\dot{\psi}=\frac{V}{L+\frac{m}{L}\left(\frac{b}{C_{\alpha F}}-\frac{a}{C_{\alpha R}}\right) V^{2}} \delta
$$

where $a$ and $b$ are the distance to the centre of gravity of the front and rear axle respectively, $\delta$ is the angle of rotation of the front wheels, $L$ is the total distance between axles, $m$ is the total mass of the vehicle located in the center of gravity, $V$ is the vehicle speed and $C_{\alpha F}$ and $C_{\alpha R}$ are the lateral stiffness coefficients of the front and rear wheels, respectively.

However, for safety reasons it will be necessary to limit the value of the yaw rate reference generated. In this case, the limit has been set as follows [6].

$$
\left|\dot{\psi}_{\text {ref,max }}\right|=\left|\frac{a_{y}}{V}\right|
$$

The yaw moment of the vehicle, for an $F_{x}$, is calculated as follows [6],

$$
\begin{gathered}
M_{z, \text { Rear }}=F_{x, R R} \frac{t_{r}}{2}+F_{x, R L} \frac{t_{r}}{2} \\
F_{x, R L}=F_{x, R R}=F_{x, R} \\
F_{x, R}=\frac{M_{z, \text { Rear }}}{t_{r}}
\end{gathered}
$$

Finally, the maximum moment in the vertical axis of the vehicle that can be generated, taking into 
account that the maximum torque that can transmit each tire is $2450 \mathrm{Nm}$.

$$
M_{z, \max }=F_{x, R, \max } t_{r}=2722 \mathrm{Nm}
$$

\section{FUZZY LOGIC CONTROLLER}

In this section a novel fuzzy controller whose objective is to control the yaw moment of the vehicle based on the yaw rate reference described in the previous section is presented. The proposed approach allows to increase and ensure the stability of the vehicle, in addition to improve its handling. Furthermore, the proposed approach, with its Multiple Input Multiple Output (MIMO) structure allows also to reduce the sideslip angle, so that both yaw rate and side slip are controlled, increasing the lateral dynamics performance of the vehicle.

\subsection{Fuzzy Logic}

Fuzzy logic is an extension of Boolean logic by Lot Zadeh in 1965 [19] based on the mathematical theory of fuzzy sets. By introducing the notion of degree in the verification of a condition, fuzzy logic provides a very valuable flexibility for reasoning [5].

The most common fuzzy logic systems structure is shown in Fig. 2. First a fuzzyfication process must be carried out to transform the input data into fuzzy sets. Second, the data is processed with an inference system, based on the developed rules. Finally, as the real world is continuous, a defuzzyfication method is necessary in order to convert the result given by the inference system into the exact value to, in our case, ensure a appropriated control.

A very important characteristic of fuzzy logic is that the rules are set in natural language in order to formalize human reasoning. In addition, the whole system is based on the designer's knowledge.

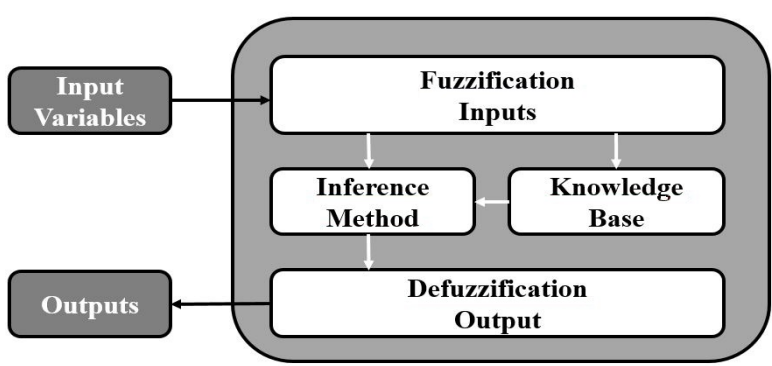

Figure 2: Fuzzy system general scheme

\subsection{Fuzzy Controller Design}

The proposed Torque Vectoring controller calculates the torque percentage to be substracted and added to each rear motor (left or right) to perform the torque distribution in the rear axle. This is, if the driver asks for a given torque $\tau$, depending on the yaw rate and side slip angle, the controller calculates a compensation torque percentage $\tau_{c}$ so that $\tau_{\text {left }}=\tau+\tau_{c}$ and $\tau_{\text {right }}=\tau-\tau_{c}$.

For that purpose the controller requires three inputs: the yaw rate error, its derivative and the side slip angle error. The yaw rate error and its derivative are calculated considering the reference detailed in the previous section. The slip angle error is calculated considering that the reference sideslip angle of the vehicle will be zero, in order to reduce the real value and obtain a neutral handling.

One of the most advantages of Fuzzy Logic is the model's abstraction at the time of tuning. This way, a distribution of 5 membership functions has been chosen for the yaw rate, e $(\psi)$, and lateral slip angle, e $(\beta)$ and three membership functions for the input of the derivative of yaw rate error. The membership functions have been selected as trapezoidal and triangular, as they provide computationally efficient calculations maintaining acceptable smoothness on the response, suitable to be implemented in conventional automotive ECUs. On the other hand, for the output, a more extensive distribution of membership functions has been chosen, nine in this case, aiming to achieve a response as close and smooth as possible.

The structure of the developed fuzzy controller is shown in Fig. 3 with the membership functions.

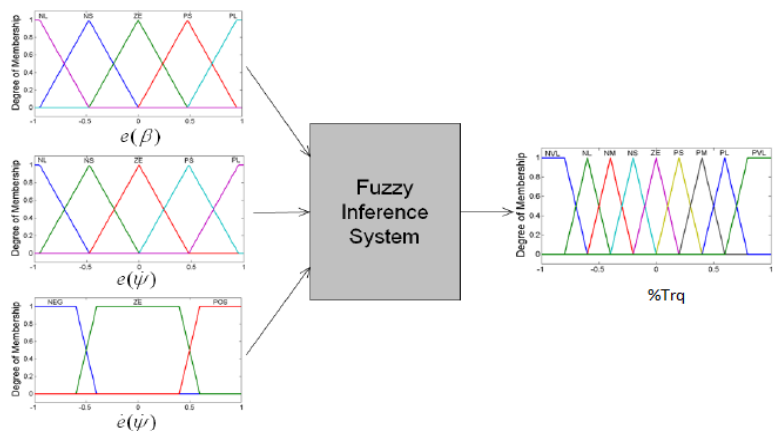

Figure 3: Fuzzy Logic Controller proposed

Subsequently the corresponding rules have been implemented based on the knowledge about the system. Table 1 shows the names and description of the membership functions, while Tables 2-4 show the implemented rules.

The control surfaces obtained are shown in Figs. 
Table 1: Membership Functions Names.

\begin{tabular}{|c|l|}
\hline Names & Description \\
\hline NVL & NEGATIVE VERY LARGE \\
\hline NL & NEGATIVE LARGE \\
\hline NM & NEGATIVE MEDIUM \\
\hline NS & NEGATIVE SMALL \\
\hline ZE & ZERO \\
\hline PS & POSITIVE SMALL \\
\hline PM & POSITIVE MEDIUM \\
\hline PL & POSITIVE LARGE \\
\hline PVL & POSITIVE VERY LARGE \\
\hline
\end{tabular}

Table 2: Rules for negative yaw rate error derivative

\begin{tabular}{|c|c|c|c|c|c|c|}
\hline$\dot{e}(\dot{\psi})<0$ & \multicolumn{6}{|c|}{$e(\dot{\psi})$} \\
\hline \multirow{1}{*}{$\mathrm{e}(\beta)$} & & NL & NS & ZE & PS & PL \\
\cline { 2 - 7 } & NL & ZE & NS & NM & NVL & NVL \\
\cline { 2 - 7 } & NS & ZE & ZE & NS & NL & NVL \\
\cline { 2 - 7 } & ZE & ZE & ZE & ZE & NS & NL \\
\cline { 2 - 7 } & PS & PS & PS & ZE & ZE & NS \\
\cline { 2 - 7 } & PL & PM & PS & PS & ZE & ZE \\
\hline
\end{tabular}

Table 3: Rules for zero yaw rate error derivative

\begin{tabular}{|c|c|c|c|c|c|c|}
\hline$\dot{e}(\dot{\psi})=0$ & \multicolumn{6}{|c|}{$e(\dot{\psi})$} \\
\hline \multirow{5}{*}{$\mathrm{e}(\beta)$} & & NL & NS & ZE & PS & PL \\
\cline { 2 - 7 } & NL & ZE & ZE & NM & NL & NVL \\
\cline { 2 - 7 } & NS & PS & ZE & NS & NM & NL \\
\cline { 2 - 7 } & ZE & PM & PS & ZE & NS & NM \\
\cline { 2 - 7 } & PS & PL & PM & PS & ZE & NS \\
\cline { 2 - 7 } & PL & PVL & PL & PM & ZE & ZE \\
\hline
\end{tabular}

Table 4: Rules for positive yaw rate error derivative

\begin{tabular}{|c|c|c|c|c|c|c|}
\hline$\dot{e}(\dot{\psi})>0$ & \multicolumn{6}{|c|}{$e(\dot{\psi})$} \\
\hline \multirow{5}{*}{$\mathrm{e}(\beta)$} & & NL & NS & ZE & PS & PL \\
\cline { 2 - 7 } & NL & ZE & ZE & NS & NS & NM \\
\cline { 2 - 7 } & NS & PS & ZE & ZE & NS & NS \\
\cline { 2 - 7 } & ZE & PL & PS & ZE & ZE & ZE \\
\cline { 2 - 7 } & PS & PVL & PL & PS & ZE & ZE \\
\cline { 2 - 7 } & PL & PVL & PVL & PM & PS & ZE \\
\hline
\end{tabular}

4 and 5. The resulting shape of each surface is uniform providing the desired smoothness in the response, in order to achieve better vehicle stability.

\section{VALIDATION}

For the validation of the proposed algorithm a commercial vehicle dynamics simulation platform, Dynacar, provided by Tecnalia Research \& Innovation, has been used [8]. This tool uses an accurate multibody formulation [2], permits the set

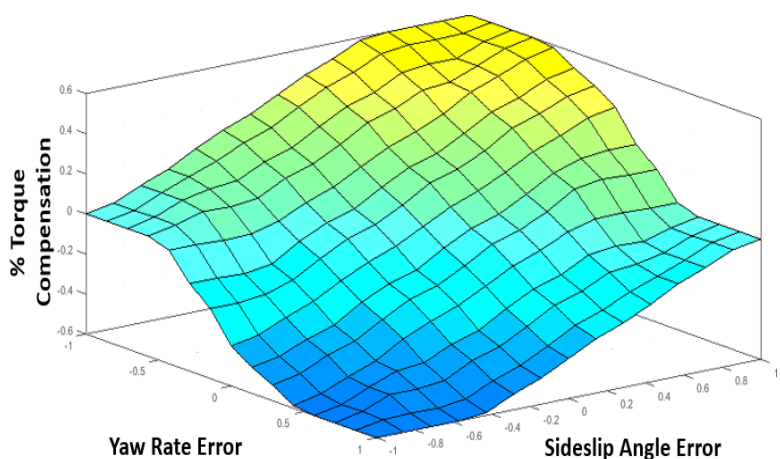

Figure 4: Control surface

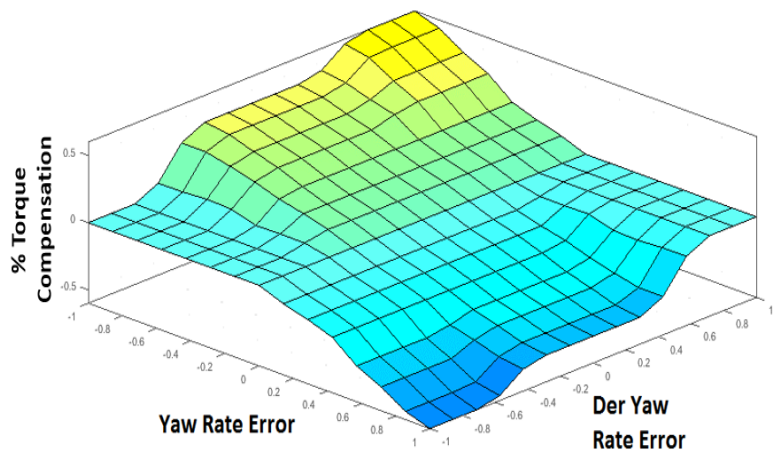

Figure 5: Control surface

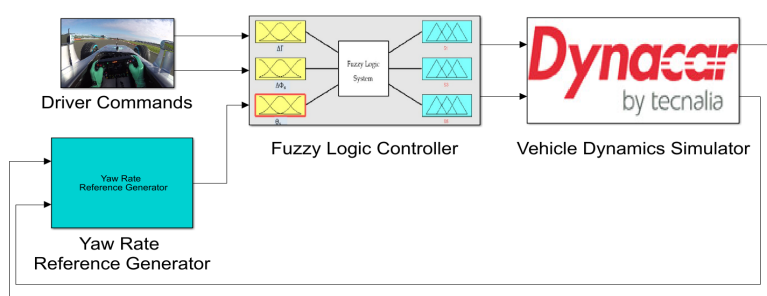

Figure 6: System general scheme

up of different MiL and HiL frameworks for accelerated time and real time simulations and has also been involved in race-track validation activities [4] [14]. The multibody model is integrated in the Matlab-Simulink framework as C-Code in an S-Function running at a sampling time of $1 \mathrm{~ms}$. Tires are modeled using an implementation of the Pacejka "Magic Formula" model, extensively used by most car manufacturers as an industry standard for vehicle model simulations [13].

The simulated vehicle is a Class E type with two independently controlled electric motors in the rear axle, which are able to provide each a power of $200 \mathrm{~kW}$ and $2450 \mathrm{Nm}$ at the wheel (after the reduction gearing).

The following table summarizes the principal char- 
acteristics of the vehicle.

Table 5: Vehicle Principal Characteristics.

\begin{tabular}{|c|l|}
\hline Mass $[\mathrm{kg}]$ & 1830 \\
\hline $\mathrm{Ix}\left[\mathrm{kgm}^{2}\right]$ & 928.1 \\
\hline $\mathrm{Iy}\left[\mathrm{kgm}^{2}\right]$ & 2788.5 \\
\hline $\mathrm{Iz}\left[\mathrm{kgm}^{2}\right]$ & 3234.0 \\
\hline Wheelbase $[\mathrm{m}]$ & 3.05 \\
\hline Front Axis Track $[\mathrm{m}]$ & 1.6 \\
\hline Rear Axis Track $[\mathrm{m}]$ & 1.6 \\
\hline
\end{tabular}

The proposed simulation setup is detailed in Fig. 6 . In order to test the previously presented control approach, a set of steering and torque commands have been programmed on the simulator, and the performance of the vehicle has been analyzed in two tests: constant curve trajectory and single lane change. Results for each will be analyzed next.

\subsection{CONSTANT CURVE TRAJECTORY}

In this test, a constant steering input of 45 degrees is applied while the vehicle keeps accelerating $0 \mathrm{~km} / \mathrm{h}$ to $80 \mathrm{~km} / \mathrm{h}$ with a constant torque request of $1500 \mathrm{Nm}$ to both wheels in total. The simulation results for both cases (with and without torque vectoring) are shown in Fig. 7 to 10 . Fig. 7 shows the trajectory of the vehicle. Fig. 8 shows the motor torques applied in both cases (without torque vectoring, same torque for both). Figs. 9 and 10 show the yaw rate and the lateral acceleration for each case, respectively.

The trajectory shows that the vehicle is able to better stay in the curve as speed increases, meaning that the understeering behaviour of the vehicle, which can be identified with this test, is clearly reduced. This is also reflected in the higher lateral acceleration and yaw-rate in Figs. 9 and 10, which shows higher values and is close to the theoretical values.

\subsection{SINGLE LANE CHANGE}

The second test consists of an evasive manoeuvre, notably more aggressive -and critical for the stability- than the previous one. A sine wave input of 45 degrees (Fig. 11) is applied while the vehicle is accelerating with a constant torque reference. The plots and legends follow the same logic as for the previous test, besides the steering wheel input in Fig. 11 and sideslip angle in Fig. 15. Fig. 12 shows the trajectory, Fig. 13 the yaw rate and Fig. 14 lateral acceleration.

In this test, there is a major difference in what

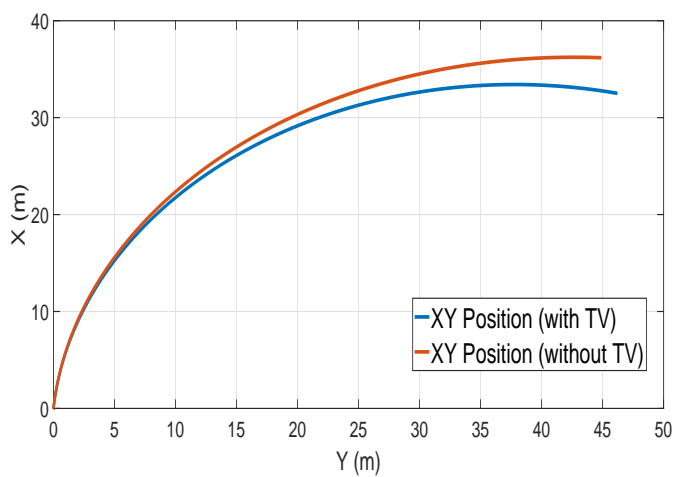

Figure 7: Trajectory

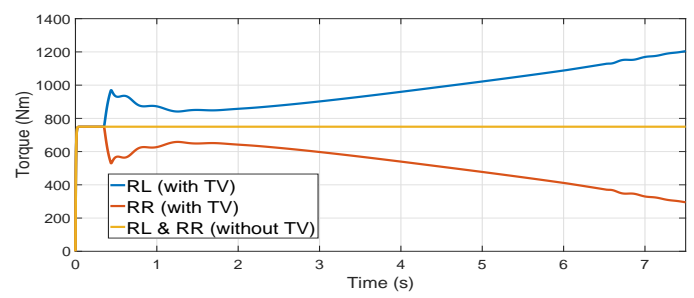

Figure 8: Wheel Torques

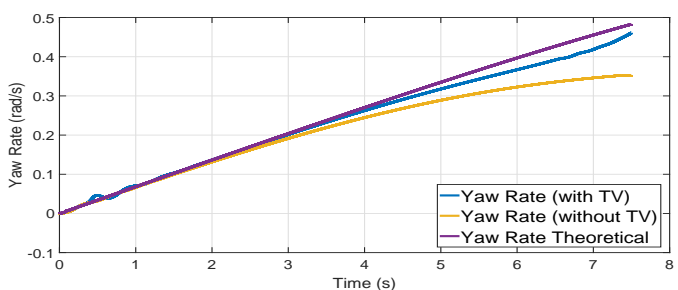

Figure 9: Yaw Rate

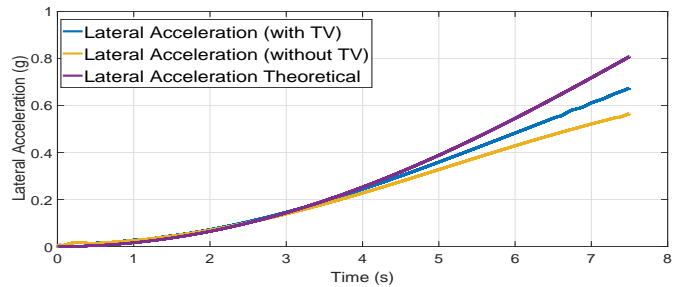

Figure 10: Lateral Acceleration

respects to the trajectory. The vehicle without torque vectoring oversteers as its rear end slides away and the vehicle ends up sliding towards the contrary side. In other words, the vehicle looses stability and it would require the presence of a skilled driver to try to regain the control over the situation. This is also reflected in the lateral acceleration and yaw rate, both reaching excessive magnitudes as the car spins and slides sideways without vectoring (the saturated value of the lateral acceleration can be seen around second 11 in Fig. 13). 
This is not the case when the torque vectoring is active. In this case the car is able to follow a neutral trajectory without loosing stability, even without a driver closing the loop. This means that the fuzzy control strategy is working correctly. It is able to generate some additional yaw rate and acceleration in the first turn, meaning that it will make a sharper turn, closer to the ideal path, without understeer, similarly to the first test. But in the second part of the manoeuvre, when it needs to turn in the opposite direction, the car tends to reach excessive yaw rate. As the fuzzy torque vectoring controller determines the theoretical yawrate internally, it takes action to follow this internal reference avoiding excessive yaw-rate while also minimizing the slip angle.

In other words, the fuzzy torque vectoring controller is able to tackle understeer in the first steering wheel movement, and then control the oversteer in the second steering movement, which otherwise leads to a dangerous stability loss.

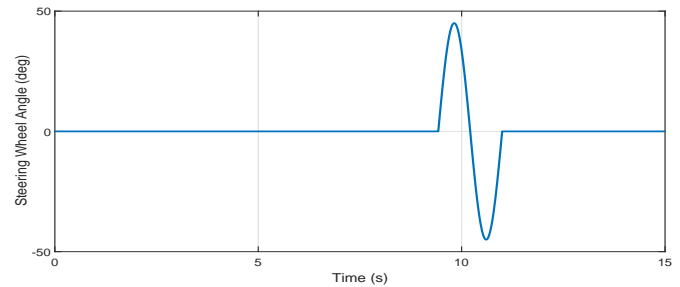

Figure 11: Steering wheel input

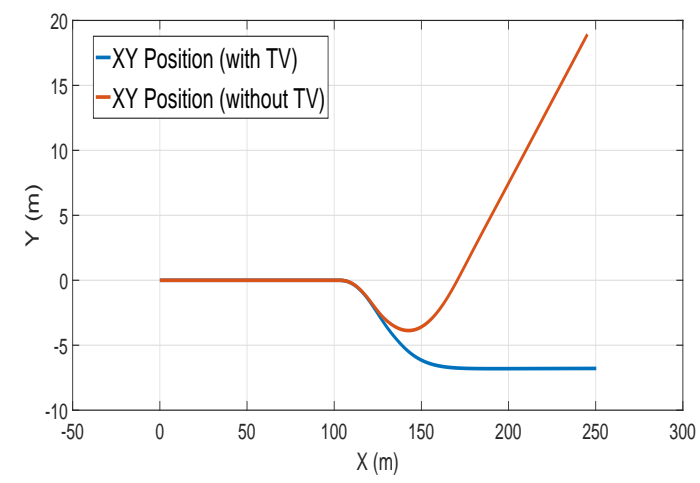

Figure 12: Trajectory

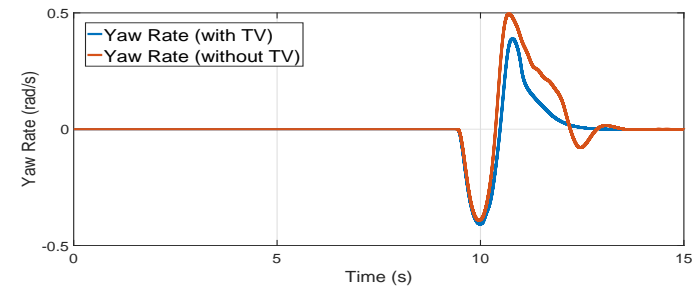

Figure 13: Yaw Rate

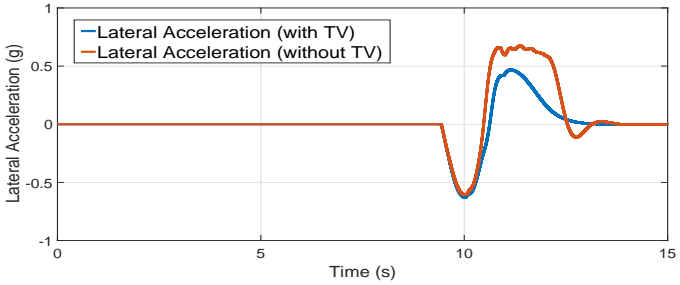

Figure 14: Lateral Acceleration

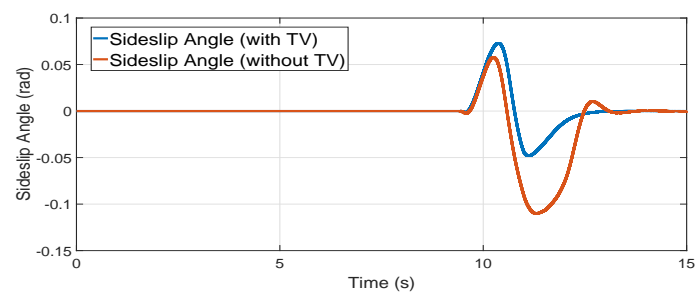

Figure 15: Sideslip angle

\section{CONCLUSIONS}

This paper has presented a novel Fuzzy Torque Vectoring Controller, for a vehicle with independent rear-wheel motors, with the objectives of increasing cornering performance while also providing additional stability. The results have been notably satisfactory, as it has shown to fulfill these typically contradictory objectives: it was able to increase the cornering forces (reducing understeer), but also was able to control the oversteer in a situation that, in absence of the controller, leads to loss of stability. This is achieved by tracking two inputs of the Fuzzy algorithm -the yaw rate and the side slip angle- while calculating an ideal yaw-rate reference in real-time.

The developed solution has been validated using a highly representative setup including a multibody vehicle dynamics simulator, which has been exploited to simulate several test procedures.

In conclusion the controllability and degrees of freedom of independent electric motors on vehicles enable enhanced dynamic handling and offer notable research potential for advanced algorithms.

Future work will include implementing an optimized internal set-point generation, while maintaining real-time execution constraints, combining with the implementation of different estimator solutions in real-time to be used as inputs for the torque vectoring controller resulting in a more elaborate algorithm, considering the implementation in new embedded platforms exploiting its computational capacities. 


\section{References}

[1] ACEA, (2016) "European Automobile Manufacturers Association - New passenger car registrations by alternative fuel type in the European Union Q4 2015".

[2] Cuadrado, J., Vilela, D., Iglesias, I., Martín, A., Peña, A., (2013) "A Multibody Model to Assess the Effect of Automotive Motor Inwheel Configuration on Vehicle Stability and Comfort", ECCOMAS Multibody Dynamics 2013.

[3] Dendaluce, M., Allende, M., Pérez, J., Prieto, P., Martin, A., (2016) "Multi Motor Electric Powertrains: Technological Potential and Implementation of a Model Based Approach "IECON 2016.

[4] Dendaluce, M., Iglesias, I., Martín, A., Prieto, P., Peña, A., (2016) "Race-Track testing of a Torque Vectoring Algorithm on a Motorin-Wheel Car using a Model-Based Methodology with a HiL and Multibody Simulator Setup", International Conference on Intelligent Transportation Systems.

[5] Dernoncourt, F. (2013) "Introduction to fuzzy logic" Massachusetts Institute of Technology.

[6] De Novellis, L., Sorniotti, A., Gruber, P., Pennycot, A., (2014) "Comparison of Feedback Control Techniques for TorqueVectoring Control of Fully Electric Vehicles" IEEE Transactions on Vehicular Technology.

[7] De Novellis, L., Sorniotti, A., Gruber, P., Shead, L., Ivanov, V., Hoepping, V., (2012)"Torque vectoring for Electric Vehicles with Individually Controlled Motors: State-of-the-Art and Future Developments". World Electric Vehicle Journal Vol. 5.

[8] "Dynacar by Tecnalia." [Online]. Available: http://www.dynacar.es/en/home.php.

[9] Haddoun, A., et al., (2006) "Sliding mode control of EV electric differential system," Proc. ICEM, Chania, Greece.

[10] Haddoun A., M.E.H. Benbouzid, D. Diallo, R. Abdessemed, J. Ghouili, K. Srairi, (2007) "Analysis, Modeling and Neural Network Traction Control of an Electric Vehicle without Differential Gears". Electric Machines \&6 Drives Conference. .

[11] Jalali, K., Uchida, T., McPhee, J., and Lambert, S., (2009) "Integrated stability control system for electric vehicles with in-wheel motors using soft computing techniques", $S A E$ International Journal of Passenger Cars Electronic and Electrical Systems.

[12] Kahveci, H., Ibrahi, H., Ekici, M., (2013) "An electronic differential system using fuzzy logic speed controlled in-wheel brushless DC motors",Power Engineering Energy and Electrical Drives.

[13] Pacejka, H.B. "Tyre and Vehicle Dynamics", $\mathrm{BH}, 2005$.

[14] Peña, A., Iglesias, I.,Valera, J.J., Martín, A., " Development and validation of Dynacar RT software, a new integrated solution for design of electric and hybrid vehicles", EVS 26 (2012).

[15] Shibahata, Y., Shimada K., Tomari T., (1993) "Improvement of Vehicle Maneuverability by Direct Yaw Moment Control", $V e$ hicle System Dynamics, pp. 465-481

[16] Shimada, K., Shibahata, Y. (1994), "Comparison of Three Active Chassis Control Methods for Stabilizing Yaw Moments", $S A E$ Technical Paper.

[17] Siampis, E., Velenis, E., Longo, S., (2015) "Model Predictive Torque Vectoring Control for Electric Vehicles Near the Limits of Handling" European Control Conference.

[18] Wallentowitz, H., (2005) "Vertical and Lateral Dynamics of Passenger Vehicles", Automotive Technology II, Institute of Automotive Engineering, Aachen University of Technology, Germany.

[19] Zadeh, L.. (1965) 'Fuzzy Sets" Information and Control. 